Full length article

\title{
Quantification of the sulfidation extent of Mo in CoMo HDS catalyst through XPS
}

\author{
M. Bravo-Sanchez ${ }^{\mathrm{a}}$, Adolfo Romero-Galarza ${ }^{\mathrm{b}, *}$, Jorge Ramírez $^{\mathrm{c}}$, Aída Gutiérrez-Alejandre ${ }^{\mathrm{c}}$, \\ Dora Alicia Solís-Casados ${ }^{\mathrm{d}}$ \\ ${ }^{a}$ Universidad de Guadalajara, Centro Universitario de Ciencias Exactas e Ingenierías, Departamento de Física, Boulevard Marcelino García Barragán 1421, Olímpica, \\ 44430 Guadalajara, Jal., Mexico \\ ${ }^{\mathrm{b}}$ Departamento de Ingeniería Química, Facultad de Ciencias Químicas, Universidad Autónoma de Coahuila, Blvd. V. Carranza e Ing. José Cárdenas V. S/N, Saltillo, \\ Coahuila 25280, Mexico \\ ${ }^{\mathrm{c}}$ UNICAT, Depto. de Ingeniería Química, Facultad de Química, UNAM, Cd. Universitaria, 04510 Cd. de México, Mexico \\ ${ }^{\mathrm{d}}$ Universidad Autónoma del Estado de México, Centro Conjunto de Investigación en Química Sustentable, UAEM-UNAM, Km 14.5, Carretera Toluca-Atlacomulco, San \\ Cayetano, Toluca, Estado de México 50200, Mexico
}

\section{A R T I C L E I N F O}

\section{Keywords:}

XPS composition

Quantitative XPS

Active approach

SVSC background

Sulfidation extent

HDS catalysts

\begin{abstract}
A B S T R A C T
Background removal in X-ray photoelectron spectroscopy (XPS) spectra was carried out in Co-Mo hydrodesulfurization (HDS) catalysts supported alumina using traditional and recent methods. The sulfidation extent, expressed by the XPS area ratio $\mathrm{A}_{\mathrm{Mo3d}-M o S 2} / \mathrm{A}_{\mathrm{Mo3d}-\mathrm{Total}}$, which is a fundamental parameter in the performance of a hydrodesulfurization catalyst, was obtained using different methodologies. The methods include the use of the background active approach, in which the background is optimized during peak-fitting. The method allows for the use of several types of backgrounds, which proved crucial for fitting the Mo $3 \mathrm{~d}-\mathrm{S} 2 \mathrm{~s}$ and the S 2p-Si $2 \mathrm{p}$ regions. Both regions contain two overlapped elements, making fundamental a distinction between them. In these cases, a slope background subtraction was used in conjunction with the Shirley-Vegh-Salvi-Castle (SVSC) method to have a clear distinction between the different strengths of backgrounds arising from the overlapped peaks Mo 3d-S 2s and S 2p-Si 2p. From the resulting fitting, the relative percentage (\% rel.) of each species present in Mo and $\mathrm{S}$ in the catalyst CoMoAl was obtained. The results were compared with those obtained using the static (traditional) approach. With these results, the sulfidation extent, an important parameter in the performance of the HDS catalyst was determined.
\end{abstract}

\section{Introduction}

The present environmental regulations require a deep hydrodesulfurization of transportation fuels, to attain sulfur concentrations near zero ppm. To achieve such standards, it is fundamental to develop more active hydrodesulfurization (HDS) catalysts, which are able to remove the most refractory sulfur-containing molecules such as 4,6dimethyldibenzothiophene.

Sulfide catalysts of Mo promoted by Co or Ni and supported on $\gamma$ alumina are used for the hydrodesulfurization of petroleum fractions [1-3]. To develop better HDS catalysts it is necessary to achieve: (i) high dispersion of the active $\mathrm{MoS}_{2}$ phase, (ii) complete sulfidation of the molybdenum and $\mathrm{Co}(\mathrm{Ni})$ precursor oxides phases, and (iii) high extent of promotion.

As is well known, XPS is a valuable technique to characterize the catalytic surface. For HDS catalysts, it provides valuable information like sulfidation extent, dispersion of the active phase on the carrier, and information about the extent of promotion [4,5].

In this work, we will be focusing on assessing the sulfidation extent, which is defined as the fraction of the Mo $3 \mathrm{~d}$ signal associated to the active $\mathrm{MoS}_{2}$ phase to the total Mo 3d signal.

XPS is one of the few techniques available for obtaining sulfidation extent and is highly valuable due its simplicity for sample preparation. With a proper controlled environment system attached to the XPS introduction-chamber, it is possible to transfer samples from the reactor to the XPS analysis chamber without exposition to air. This capability allows obtaining accurate peak intensities without the contribution of undesirable $\mathrm{Mo}^{5+}$ formed when the catalyst is exposed to air.

Although there are numerous works focused on the evaluation of sulfidation extent by XPS [4,6-13], there is discrepancy between the

\footnotetext{
* Corresponding authors.

E-mail addresses: mariela.bravo@academicos.udg.mx (M. Bravo-Sanchez), a_romero@uadec.edu.mx (A. Romero-Galarza).
} 
Table 1

Compilation of references where are showed different methods used to certain XPS qualitative and quantitative analysis.

\begin{tabular}{|c|c|c|c|}
\hline Catalyst & Analysis & Method & Ref. \\
\hline $\mathrm{CoMo} / \mathrm{TiO}_{2}$ & Quantification CoMoS surface density & $\begin{array}{l}\text { Deconvolution: Mixed Gaussian-Lorenztian functions a nor-linear squares fitting algorithm. } \\
\text { Background subtraction: Shirley }\end{array}$ & [4] \\
\hline $\mathrm{CoMo} / \mathrm{Al}_{2} \mathrm{O}_{3}$ & Quantification of CoMoS, and $\mathrm{MoS}_{2}$ phases & $\begin{array}{l}\text { The spectra were integrated by applying a Shirley type baseline. } \\
\text { Software: The spectra were analysed by using CasaXPS V. } 2.0 .71\end{array}$ & [5] \\
\hline $\mathrm{CoMo} / \mathrm{Al}_{2} \mathrm{O}_{3}$ & The degree of sulfidation of Co and Mo & $\begin{array}{l}\text { Deconvolution: The spectra were fitted by Gaussian (65\%)-Lorentzian (35\%). The spectra } \\
\text { were fitted using XPSCasa software. } \\
\text { A Shirley background subtraction was applied. }\end{array}$ & [7] \\
\hline $\mathrm{Co}(\mathrm{Ni}) / \mathrm{Al}_{2} \mathrm{O}_{3}$ & $\begin{array}{l}\text { Qualitative determination of } \mathrm{MoS}_{2} \text { and } \mathrm{Co}(\mathrm{Ni}) \mathrm{MoS} \\
\text { phases }\end{array}$ & $\begin{array}{l}\text { The deconvolution of the spectra was made using mixed Gaussian Lorentzian functions with } \\
\text { an iterative least-squares computer program. }\end{array}$ & [11] \\
\hline $\mathrm{NiMoP} / \mathrm{Al}_{2} \mathrm{O}_{3}$ & Promotor ratio $\mathrm{NiMoS} / \mathrm{Mo}^{4+}$ & $\begin{array}{l}\text { Experimental peaks were decomposed into components using mixed Gaussian-Lorentzian } \\
\text { functions and a nor-linear squares fitting algorithm. } \\
\text { Shirley background susbtraction was applied }\end{array}$ & [12] \\
\hline $\mathrm{NiMo} / \mathrm{SiO}_{2}-\mathrm{Al}_{2} \mathrm{O}_{3}$ & $\begin{array}{l}\text { Quantification of sulfidation extent and the NiMoS } \\
\text { phase of the catalyst. }\end{array}$ & $\begin{array}{l}\text { The spectra were fitted to a Shirley- Linear background using XPSPEAK version } 4.1 \text { software. } \\
\text { The deconvolution of Mo 3d was completed by the mix Gaussian-Lorentzian functions. }\end{array}$ & [13] \\
\hline
\end{tabular}

methods used for analysis as is showed in the Table 1.

The spectra of the relevant core levels considered for this study (i.e. Mo 3d-S 2s and S 2p-Si 2p) are particularly challenging because of overlapping of peaks with different background behaviour. For such cases, it is crucial to reveal the true peak areas that correspond to each core level. Efforts from other authors have provided useful information about the decomposition of the Mo 3d-S 2s region, unveiling the positions of the $\mathrm{S} 2 \mathrm{~s}$ and Mo $3 \mathrm{~d}$ components in the sulfide catalyst, though without emphasis on differentiating the origin of the different backgrounds on that region [11]. Moreover, assessment of the stoichiometry has barely been reported. To our knowledge, only one publication has been devoted to this issue [14]. However, the background was not addressed considering their different origin and the method used in that work involves the use of reference samples, which in practical data acquisition are not usually available. Certainly, in [14] it is mentioned the necessity of a quantitative analysis, since it is fundamental to calculate with accuracy the sulfidation extent to show the effective formation of $\mathrm{MoS}_{2}$ from the oxide, as will be performed in this work.

The efficiency of the methods SVSC, and slope under active approach that we used, have been successfully demonstrated by HerreraGomez et al. $[15,16]$ in well controlled synthetized samples, where quality of data is not a factor that concern for fitting purposes, since the intensity of peaks is quite clear. It is worthy to note that in the case of HDS catalysts supported based on CoMo generally the concentrations are low. Usually the HDS catalysts were prepared with a Mo surface concentration of $2.8 \mathrm{Mo}$ atoms $/ \mathrm{nm}^{2}$ and $\mathrm{Co} / \mathrm{Co}+\mathrm{Mo}$ of 0.33 , which correspond to $8.124 \mathrm{wt} \% \mathrm{Mo}$, and $3.981 \mathrm{wt} \%$ Co. At such concentrations, the intensity of the photoemitted signal is low for the relevant elements, making complicated the proper identification of chemical states. We believe that the application of these methods on problems, as catalysis, where low concentrations of the elements at stake are low, will help to unveil proper insights in the development of these materials.

With this study, we expect to provide valuable information by showing the advantages of carrying out a quantitative analysis applying methods of background removal: Shirley-Vegh-Salvi-Castle (SVSC) and slope background under active approach.

\section{Samples and methods}

\subsection{Catalyst preparation}

The $\mathrm{CoMo} / \mathrm{Al}_{2} \mathrm{O}_{3}$ catalyst was prepared conventionally by pore volume co-impregnation of a commercial $\gamma$-alumina (Alumina 2.5/210, SASOL) support by aqueous solutions of cobalt nitrate $\left(\left(\mathrm{CoNO}_{3}\right)_{2} * 6 \mathrm{H}_{2} \mathrm{O}\right.$ $98 \%$, Aldrich), and ammonium heptamolybdate $\left(\left(\mathrm{NH}_{4}\right)_{6} \mathrm{Mo}_{7} \mathrm{O}_{24} * 4 \mathrm{H}_{2} \mathrm{O}\right.$, 97\% Aldrich). The commercial $\gamma$-alumina contains $0.12 \mathrm{ppm}$ of $\mathrm{SiO}_{2}$. The catalyst was prepared with a Mo surface concentration of 2.8 atoms of $\mathrm{Mo} / \mathrm{nm}^{2}$, and the necessary amount of Co to obtain an atomic Co/ (Co $+\mathrm{Mo}$ ) ratio of 0.33 . After impregnation the catalyst was dried for $12 \mathrm{~h}$ at $373 \mathrm{~K}$ and calcined at $673 \mathrm{~K}$ during $4 \mathrm{~h}$. The sulfidation procedure was carried out in a continuous flow reactor using $20 \mathrm{~mL} / \mathrm{min}$ of a $\mathrm{H}_{2} \mathrm{~S}(15 \mathrm{vol} \%) / \mathrm{H}_{2}$ gas mixture at $400{ }^{\circ} \mathrm{C}$ during $4 \mathrm{~h}$, with a heating ramp of $5{ }^{\circ} \mathrm{C} / \mathrm{min}$. Hereafter, the Co promoted catalyst will be named CoMoAl.

\subsection{Methods of fitting}

XPS data processing, including background removal and deconvolution of peaks, was performed using an interactive least-squares computer program, AAnalyzer ${ }^{\circledR}$ version 1.2 [17].

For background removal, two types of approaches were applied: static (traditional) and active (recently reported). The static approach is called in this way since the background is defined before the peak fitting. The user must guarantee, by selecting a region between two energy points, that all the contribution to the photoemission is inside the region selected. This method often conveys underestimation of areas since beyond the region selected it could be an additional contribution to the photoemission signal. In general, the typical models applied under this approach comprehend Shirley, Tougaard and linear backgrounds methods, which are applied in an independent way depending on the characteristics of the spectra. This is, only one type of method can be applied to the entire spectra, giving one value for the background. In this work, for comparison purposes we have selected Shirley background to use under the static approach, since it is the most common.

In contrast, in the active approach, the background and intensity are optimized during fitting, allowing to consider the contribution of photoemission near the region and making the selection of ends points of the region independent to the user. When the active approach is used, it is possible to use more than one type of background at the same time, which can be present in the spectra due the extrinsic or intrinsic energy losses [18]. It is important to note that in the case of extrinsic scattering, it has been demonstrated that the background originated by this effect, can be modelled accurately using slope background [15], while the intrinsic effect due the photoexcitation process can be modelled using SVSC or Shirley. The selection of these two last methods is made depending if the spectrum has (SVSC) or not (Shirley) overlapping with other core levels, in which case, is used the SVSC or Shirley, respectively. In our case of study, SVSC has been used since both core levels of interest, Mo $3 \mathrm{~d}$ and $S \mathrm{p}$ are overlapped with components from other core levels. The selection of proper contributions to the background is favourable for quantification, as will be shown.

We will show the scope of the two approaches, mainly in: (i) the dependence of the selected range for the analysis (different limits at 


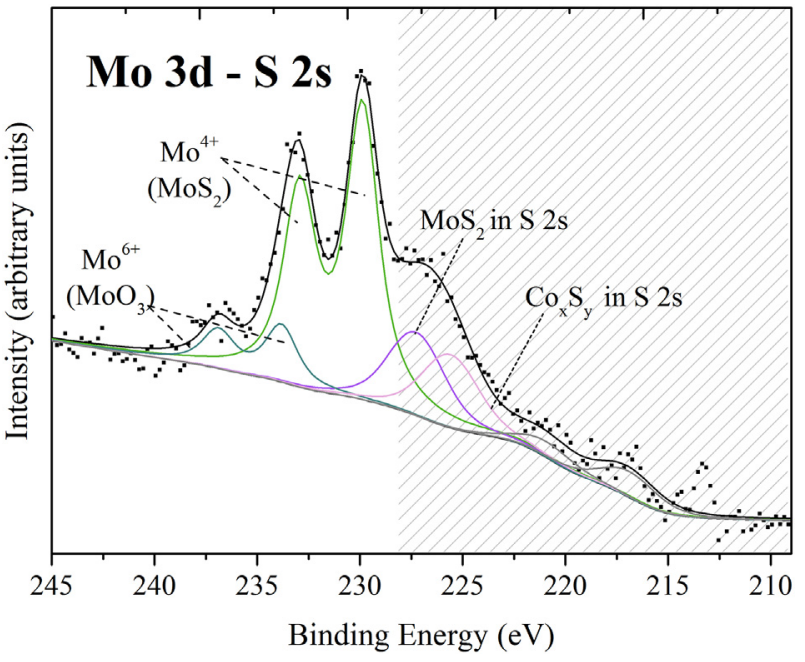

Fig. 1. Mo 3d-S 2s spectra of the sulfided catalysts showing the corresponded S 2 s components in the Mo $3 d-S 2 s$ region (right side of spectra with line pattern). The area of $\mathrm{MoS}_{2}$ component in S 2s is related with the area of the $\mathrm{MoS}_{2}$ component in $S 2$ p, by a ratio $S 2 \mathrm{~s} / \mathrm{S} 2 \mathrm{p}$ of 0.65 .

high binding energy) over the areas obtained. (ii) The quality of the fitting (chi-square, $\chi^{2}$ ) in conjunction with (iii) the stoichiometry of the $\mathrm{MoS}_{2}$ specie obtained in coaction with well-known detailed methods of quantification (using physic parameters) [19].

\subsection{Method for composition assessment}

The stoichiometry was assessed using physical parameters involved in the creation of the photoemission signal: the photoelectric differential photoionization cross section of the atom $\left(\frac{\delta \sigma_{c A}}{\delta \Omega}\right)$, which was evaluated considering the correction for the effect of monochromator as described elsewhere [20] and the effective attenuation length $\left(\lambda_{A}\right)$ that was calculated using NIST Electron Effective-Attenuation-Length Database Version 1.3. In Eq. (1) is given the relationship between the atomic density of an element $A$ ( $N_{A}$, proportional to its stoichiometry), the intensity of the photoemission spectra $\left(I_{A}\right)$, the photoelectron kinetic energy $K E_{A}$, and the physical parameters $\frac{\delta \sigma_{c A}}{\delta \Omega}$ and $\lambda_{A}$.

$N_{A} \sim \frac{I_{A} K E}{\frac{d \sigma_{c A}}{d \Omega} \lambda_{A}}$

The value $I_{A}$ corresponds to the area of the photoemission signal obtained after the fitting of the core levels Mo 3d, S 2p, O 1s, and Al 2p, associated to an element $A$.

\subsection{XPS data acquisition}

The system used for XPS data acquisition was an instrument assembled by Intercovamex (Morelos, Mexico) equipped with seven channel Alpha110 hemispherical spectrometer from ThermoFisher (East Grinstead, UK), working with a monochromatic Al-Ka source with photon energy of $1486.7 \mathrm{eV}$ at take-off angle of $41^{\circ}, 500 \mu \mathrm{m}$ size beam, and operating at $1.2 \times 10^{-9}$ Torr. This system has a glove box attached to the prechamber, which allows the transferring of the samples to the system without exposing them to the oxygen in the environment. Due to the non-conductive nature of the samples, a flood gun for charging compensation was used during data acquisition.

The sulfided catalyst sample CoMoAl was transported under inert atmosphere and placed into the prechamber through the glove box. Once there, it was outgassed at $3 \times 10^{-7}$ Torr for $3 \mathrm{~h}$. After this time, the sample was transferred to the analysis chamber. The analysis began with survey acquisition at $50 \mathrm{eV}$ of pass energy and $1 \mathrm{eV}$ of step size, followed by high-resolution scans of the regions Mo 3d-S 2s, S 2p-Si 2p,
$\mathrm{O} 1 \mathrm{~s}$, and $\mathrm{Al} 2 \mathrm{p}$, at $15 \mathrm{eV}$ of pass energy and a step size of $0.1 \mathrm{eV}$.

\section{Results and discussion}

The high-resolution spectra of the core levels involved in the sulfurization extent (i.e. Mo 3d and S 2p) are described in terms of their fitting parameters (i.e. binding energy, Gaussian and Lorentzian values) using the described methods for background removal. Using the resulting areas from regions Mo $3 \mathrm{~d}$ and S $2 \mathrm{p}$, the stoichiometry was calculated for the $\mathrm{MoS}_{2}$. Furthermore, considering that the catalyst is supported on $\gamma$-alumina, the stoichiometry of $\mathrm{Al}_{2} \mathrm{O}_{3}$ was calculated to provide an accurate reference for the shift correction in all the spectra. The value of binding energy (BE) used for this purpose was $74.1 \mathrm{eV}$ (Al 2p) [21].

Afterwards, a comparison of the methods used for background removal (i.e. active vs. static approaches) are illustrated in detail. Finally, the sulfidation extent obtained with both methods compared is given, making emphasis on the values obtained using SCSV background plus slope under the active approach.

\subsection{Fitting of spectra}

\subsubsection{Mo-species}

In Fig. 1, it can be observed that the components of the core level Mo 3d overlap strongly with S 2 s region. To obtain the real contribution of areas for quantification of molybdenum, a clear distinction of the background of the components for each core level was done using the SVSC background under active approach.

In this case, it is crucial to remark the importance of using SVSC background on this overlapped Mo 3d-S 2s spectra, since assigning an improper strength of background for the different elements in the spectrum generally leads to an underestimation of the peak area, although overestimations of areas are not discarded. The assignations of different values of background for each component is direct using the software AAnalyzer ${ }^{\circledR}$, where the values of SVSC backgrounds for each component are assigned by the software according to the characteristics of the spectra (free values) in the same way as the areas were obtained (including the area of $\mathrm{S} 2 \mathrm{~s}$ from $\mathrm{MoS}_{2}$ component). In addition, a slope background due to the change in the tendency of the background at high $\mathrm{BE}$ near the region was required.

The two doublets found for Mo $3 \mathrm{~d}$ were fitted with the characteristic separation of $3.2 \mathrm{eV}$ and ratio between spin-orbit doublet of 0.66 . Based on the positions of branch $3 \mathrm{~d}_{5 / 2}$, the peak at $229.8 \mathrm{eV}$ in CoMoAl is associated to $\mathrm{Mo}^{4+}$, which is characteristic of molybdenum disulfide $\left(\mathrm{MoS}_{2}\right)$. The peaks at $233.8 \mathrm{eV}$ is associated to $\mathrm{Mo}^{6+}$, characteristic of molybdenum oxide $\left(\mathrm{MoO}_{3}\right)$.

A convolution of Gaussian and Lorentzian (Voigt line-shape) was employed for all the peaks. The values for Gaussian and Lorentzian were correlated to the same value between components of the same core level. During the fitting, binding energies and areas took free values. Table 2 summarizes the fitting values for all the core levels and their components, obtained using the backgrounds SVSC plus slope.

\subsubsection{Sulfur species}

The core level S 2p was analysed, since its probability of photoemission is higher than the $S 2 \mathrm{~s}$, and the peak is narrower, allowing to accurately determine the chemical states present in the signal. The $S 2 p$ core level is overlapped at lower binding energy with photoemission from silicon (present in the commercial alumina) and is crucial to unveil clearly the components in the spectra, principally the contribution of the $\mathrm{MoS}_{2}$. The fitting of this region was performed using the SVSC background and the slope background under the active approach, as was performed for the region Mo $3 \mathrm{~d}-\mathrm{S} 2 \mathrm{~s}$. The $\mathrm{S} 2 \mathrm{p}$ spectra are composed of the spin-orbit doublet $S 2 p_{3 / 2}$ and $S 2 p_{1 / 2}$ with a ratio of 0.5 and splitting of 1.18 between them. The doublets are not resolved due the resolution of the instrument, and instead, a single asymmetric peak 
Table 2

Values of binding energies, Gaussian and Lorentzian are given for the fitting obtained with the background SVSC + slope. These Gaussian and Lorentzian are correlated to the same value between the correspondent branches $5 / 2$ and $3 / 2$ and between the components of the same core level. The relative percentage of each specie was obtained dividing its peak area by the total area formed by the different species of the same element present in the sample.

\begin{tabular}{|c|c|c|c|c|c|}
\hline \multirow[b]{2}{*}{ Region } & \multirow[b]{2}{*}{ Peak } & \multicolumn{4}{|c|}{ CoMoAl } \\
\hline & & $\begin{array}{l}\mathrm{BE} \\
\mathrm{eV}\end{array}$ & $\begin{array}{l}\text { Gaussian } \\
\mathrm{eV}\end{array}$ & $\begin{array}{l}\text { Lorentzian } \\
\text { eV }\end{array}$ & $\%$ rel. \\
\hline \multirow[t]{2}{*}{ Mo 3d } & $\mathrm{MoS}_{2}$ & 229.8 & 0.7 & 1.57 & 83.8 \\
\hline & $\mathrm{MoO}_{3}$ & 233.8 & 0.7 & 1.57 & 16.2 \\
\hline \multirow[t]{3}{*}{ S 2p } & $\mathrm{MoS}_{2}$ & 162.8 & 0.83 & 1.2 & 75 \\
\hline & $\mathrm{Co}_{\mathrm{x}} \mathrm{S}_{\mathrm{y}}$ & 160.2 & 0.83 & 1.2 & 17.4 \\
\hline & $\mathrm{CoSO}_{4}$ & 171.2 & 0.83 & 1.2 & 7.5 \\
\hline \multirow[t]{2}{*}{$\mathrm{Al} \mathrm{2p}$} & $\gamma$-Alumina & 74.1 & 1.89 & 0.2 & 17.9 \\
\hline & Hydroxides/oxy-hydroxides & 75.6 & 1.89 & 0.2 & 82.1 \\
\hline \multirow[t]{3}{*}{$\mathrm{O} 1 \mathrm{~s}$} & $\gamma$-Alumina & 530.2 & 2.0 & 0.27 & 15.0 \\
\hline & Hydroxides/oxy-hydroxides & 531.9 & 2.0 & 0.27 & 55.6 \\
\hline & $\mathrm{SiO}_{2}$ & 532.9 & 2.0 & 0.27 & 29.4 \\
\hline
\end{tabular}

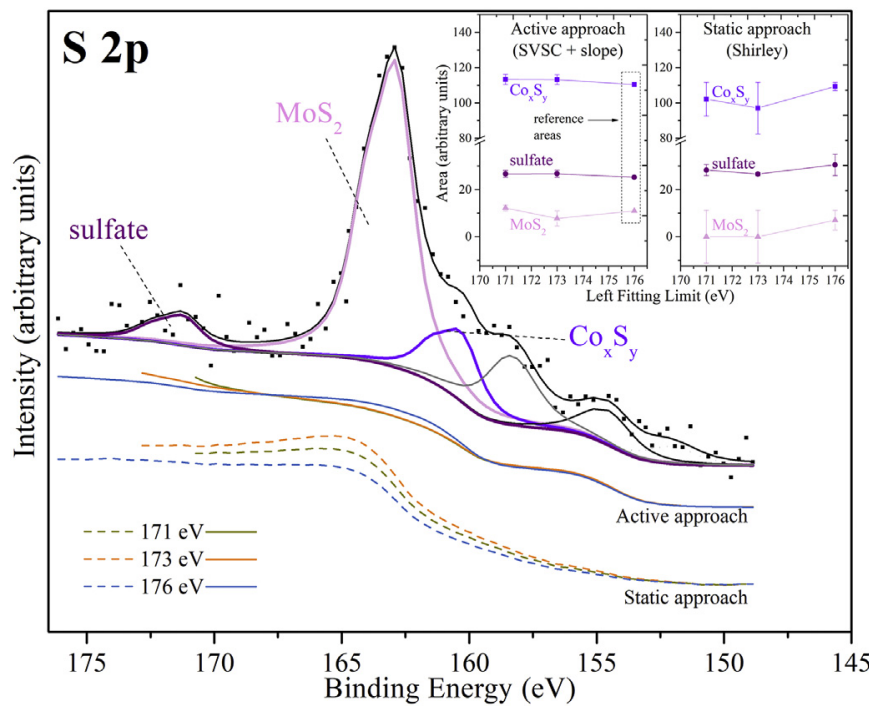

Fig. 2. S 2p spectrum of the sulfided catalysts CoMoAl. Inset shows the dependence of the resultant area on the selection of range with the use of both, static and active approaches. For error calculation, the areas used as references were those with which were obtained the closest values for stoichiometry $\mathrm{MoS}_{2}$ (under active approach).

is visible (see Fig. 2). The spectrum is described based on the $\mathrm{BE}$ of the branch S $2 \mathrm{p}_{3 / 2}$. The peak at $160.2 \mathrm{eV}$ corresponds to sulfur from sulfide phase $\mathrm{Co}_{\mathrm{x}} \mathrm{S}_{\mathrm{y}}$. The peak at $162.8 \mathrm{eV}$ is ascribed to $\mathrm{S}^{2-}$ of the $\mathrm{MoS}_{2}$ phase [11]. A doublet at $171.2 \mathrm{eV}$ is assigned to sulfur from sulfate $\mathrm{CoSO}_{4}$ which can be the result of a slight oxidation during the handling of the sample. Two additional peaks were found at low binding energy, which belong to the region of the core level Si 2 s.

\subsubsection{Aluminium and oxygen species}

The line-shape in the regions $\mathrm{Al} 2 \mathrm{p}$ and $\mathrm{O} 1 \mathrm{~s}$ was modelled using Voigt, and for background removal the slope was used. Two chemical states for $\mathrm{Al} 2 \mathrm{p}$ were found: $\mathrm{Al}$ in crystal structure of $\gamma$-alumina at $74.1 \mathrm{eV}$ associated to $\mathrm{Al}_{2} \mathrm{O}_{3}$, and $\mathrm{Al}$ in hydroxides/oxyhydroxides at $75.6 \mathrm{eV}$. In $\mathrm{O} 1 \mathrm{~s}$ three chemical states were found: $\mathrm{O}$ in crystal structure of $\gamma$-alumina at a position of $529.9 \mathrm{eV}$, confirming $\mathrm{Al}_{2} \mathrm{O}_{3}, \mathrm{O}$ in hydroxides/oxyhydroxides at $531.5 \mathrm{eV}$. A third peak in $\mathrm{O} 1 \mathrm{~s}$ is assigned to silicon at a BE of $532.4 \mathrm{eV}$. These values are in strong agreement with literature [21-24].

\subsection{Stoichiometry}

The density of elements $A\left(N_{A}\right)$ present in each particular sample was obtained with Eq. (1). The stoichiometric formula (atomic ratio, $a_{r}$ ) was obtained using $R_{A}{ }^{a_{r}}=\left(N_{A} / N_{B}\right) \cdot n_{B}$, where $N_{B}$ and $n_{B}$ are the concentration and the number of atoms of a particular element and the selected base element, respectively. The assessment of stoichiometry gives the next results: $\mathrm{Mo}_{0.995} \mathrm{~S}_{2}$ and $\mathrm{Al}_{2.06} \mathrm{O}_{3}$.

\subsection{Comparison of methods}

For S 2p and Mo 3d, the fitting areas and stoichiometry obtained with both approaches has been compared. In the case of $S 2 p$, two types of background were compared: Shirley background under static approach and the SVSC plus slope under the active approach. These were applied at three different limits at high binding energy (left of the peak): $176 \mathrm{eV}, 173 \mathrm{eV}$, and $171 \mathrm{eV}$. The two types of background applied under both approaches at those ranges are shown below the fitted spectra in Fig. 2. The set of background lines at three different ranges for the active approach shows variation in their relative intensity as small as $2.5 \%$ for the peak $\mathrm{MoS}_{2}$ for the limit range $171 \mathrm{eV}$. The bigger variation was found to be $30 \%$ for the sulfate peak when the limit used was $173 \mathrm{eV}$. In the case of the set of background lines under the static approach it is possible to observe at first glance more variation in intensities. With the highest value being $100 \%$ for the sulfate peak at $173 \mathrm{eV}$ and $171 \mathrm{eV}$. In the case of the peak involved in the sulfidation assessment $\mathrm{MoS}_{2}$, when active approach is used the area variations respecting the area obtained at $176 \mathrm{eV}$ are $2.5 \%$ and $2.6 \%$, for $173 \mathrm{eV}$ and $171 \mathrm{eV}$, respectively. With the use of static approach, the variations in areas are $12.0 \%$ and $7.0 \%$ for $173 \mathrm{eV}$ and $171 \mathrm{eV}$, respectively. Observe that using either active or static approach at the left limit of $176 \mathrm{eV}$, the backgrounds look almost the same (continue and dotted blue lines), with a variation for example, for the relevant peak for sulfidation being $1.0 \%$. In the ideal cases when the complete range was acquired it is discretional the election of the approach to be used. However, when the complete range of the spectra has not been acquired and it cannot be repeated for any reason, still is possible to rescue information from nearly missing peaks, with the use of active approach. Another point regarding the analysis of the peak with static approach if a proper range was acquired (with at least $3 \mathrm{eV}$ of flat background at each side of the photoemission peak), is the dependence of the selected range, since the area of the peaks are strongly dependant on the user selection of the end points as was proved in [16].

The resultant variation in the area of the peaks imply changes in the stoichiometry of the chemical compound present in the sample as is shown in the molybdenum spectra (Fig. 4). The inset in Fig. 2 shows the dependence of the area on the selected range for each component, for the approaches used. The reference areas of $S 2 p$ considered to calculate these variations are those with which the stoichiometry is closest to $\mathrm{MoS}_{2}$.

For $\mathrm{S} 2 \mathrm{~s}$, the error in the area can be additionally determined from the expected area ratio $S 2 s / S 2 p$, that can be assessed from the photoelectric cross section for a polarized source [20]. In this case, the peak in the $S 2 s$ and $S 2 p$ regions considered for this calculation is the component related with $\mathrm{MoS}_{2}$. From the theoretical cross section values $2 \mathrm{~s}$ and $2 \mathrm{p}$ results a ratio $2 \mathrm{~s} / 2 \mathrm{p}$ of 0.65 for sulfur, which follows the $2 \mathrm{~s}$ / $2 p$ trend for the elements from Aluminium to Germanium, according to their atomic number as the Fig. 3 displays. Qiu et al. obtained an empirical value of 0.66 from the ZnS spectra [11]. In our case, the assessment of the ratio $\mathrm{S} 2 \mathrm{p} / \mathrm{S} 2 \mathrm{~s}$ with the experimental areas obtained after fitting with SVSC and slope background gives a value of 0.76 .

When Shirley under static approach is used in both regions $\mathrm{S} 2 \mathrm{~s}$ and $S 2 p$, the value of the core levels area ratio $S 2 s / S 2 p$ is 1.4 , which is far from the expected value.

Fig. 4 illustrates the Mo $3 \mathrm{~d}$ region where are compared the same background removal methods as was compared in the $S 2 p$ spectrum 


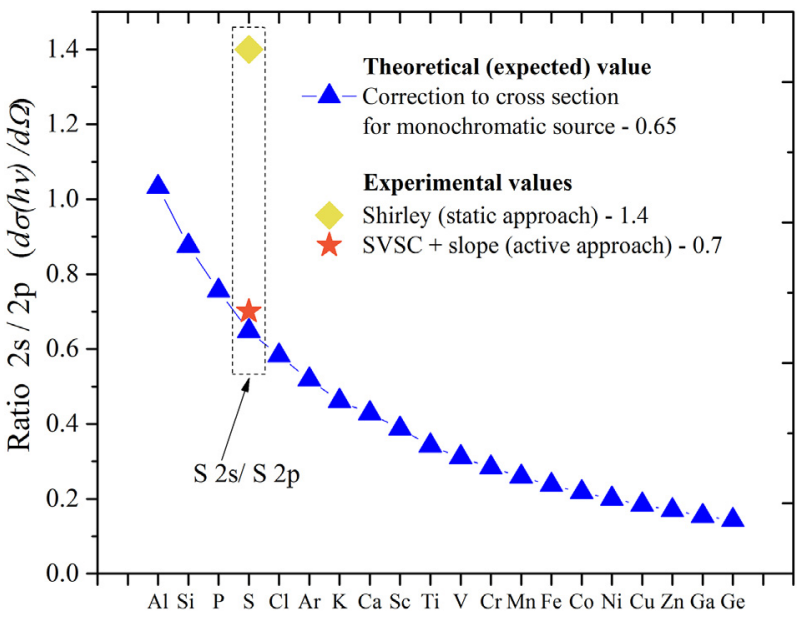

Fig. 3. Ratios of cross section $2 \mathrm{~s} / 2 \mathrm{p}$ for elements from $\mathrm{Z}=13$ to $Z=32$ evaluated from calculations of atomic subshell photoionization cross section for $S$ 2s and S 2p core levels calculated for the case of monochromatic source (polarized source) as in [20]. A value close from expected (star symbol) was found when using SVSC + slope (active approach).

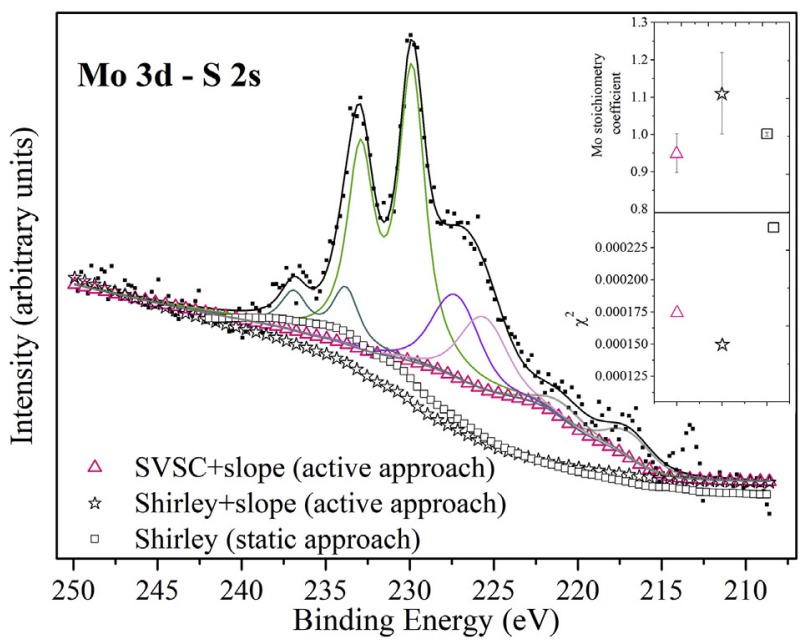

Fig. 4. a) Mo $3 d-S$ s spectrum of CoMoAl sample showing three types of removal background methods: Shirley (static approach), Shirley plus slope background subtraction (active approach), and the methods SVSC plus slope (active approach). Inset: it is shown the stoichiometric value of $\mathrm{Mo}\left(\mathrm{MoS}_{2}\right.$ as model) as function of the type of background and the chi-square value for each type of background.

(SVSC + slope, active approach and Shirley, static approach) and additionally the Shirley plus slope under active approach. As it was showed with the $S 2 p$ spectrum, the static Shirley background is strongly dependent on the selected range. In this case, a fitting at $246 \mathrm{eV}$ at the left limit entails a missing area for the $\mathrm{Mo}^{6+}$ and underestimation of areas for the rest of the components in Mo 3d. The best fitting for the static approach, was obtained at $242 \mathrm{eV}$ at the left limit of binding, with an undervalue of $29 \%$ for the total area in relation to the area obtained with the SVSC plus slope background. Nonetheless, with the areas obtained at this $\mathrm{BE}$ as left limit, the value for Mo stoichiometry was 1.06, whilst the expected value for Mo stoichiometry is one. Given that value, it is tempting to use the static approach. However, it is mandatory to consider the whole scenery, considering factors as the dependence of the area on the selected range, the fitting parameters, and the quality of the fitting evaluated for example with the chi-square as is evaluated in this work.

In the inset of Fig. 4, is shown the quality of the fitting (chi-square, $\chi^{2}$ ) and the values obtained for Mo stoichiometry in function of the three methods applied in the Mo 3d spectrum. In the three cases of background comparison, the areas of the $S 2 p$ components used for the calculations were those obtained with SVSC plus slope (active approach). For Mo stoichiometry both the Shirley (static approach) and SVSC (active approach) background present a close value to 1 , however with the static Shirley the value of $\chi^{2}$ is the highest compared with the other two backgrounds (inset of Fig. 4). This could be an acceptable result without consider the incertitude when using static Shirley due the dependence on the selected range. When Shirley plus slope is used in the active approach, the value of $\chi^{2}$ indicates a good fit, even better than for SVSC plus slope. However, the stoichiometry of Mo is not the expected, presenting an error of \pm 0.11 . This result indicates that even an active approach is used, still is necessary to distinguish between the individual contributions of the different core levels and chemical states to the final background using SVSC background. In the case of SVSC plus slope background, the method conveys an error of \pm 0.05 in stoichiometry and an acceptable value of chi-square. This result is promising, if we also consider the independence of the selected range over the areas obtained, and, as discussed above the closest value to 0.65 for the ratio $S 2 p / S 2$ s was obtained with SVSC plus slope background.

\subsection{Sulfidation extent calculation}

As mentioned in Section I1, the sulfidation extent is a relevant indicator related to the catalytic performance. This value was assessed using the approaches compared in this work. As a result, the sulfidation extent value obtained using the static approach seems to be the best (90\%), according with the ideal value equal to one, which means that almost all the $\mathrm{MoO}_{3}$ present in the sample is completely sulfided $\left(\mathrm{MoS}_{2}\right)$. However, this value is more idealized than correct and can give an improper insight for the sulfidation extent. Finally, the value obtained with the use of SVSC background plus slope for the sample was 84\% (Fig. 5).

Considering the compendium of results for stoichiometry and quality of fitting for both samples it can be determined that the best method for fitting the Mo $3 \mathrm{~d}-\mathrm{S} 2 \mathrm{~s}$ an $\mathrm{S} 2 \mathrm{p}-\mathrm{Si} 2 \mathrm{p}$ regions and therefore for calculating the sulfidation extent, it is the mix of removal background methods SVSC + slope applied under the active approach.

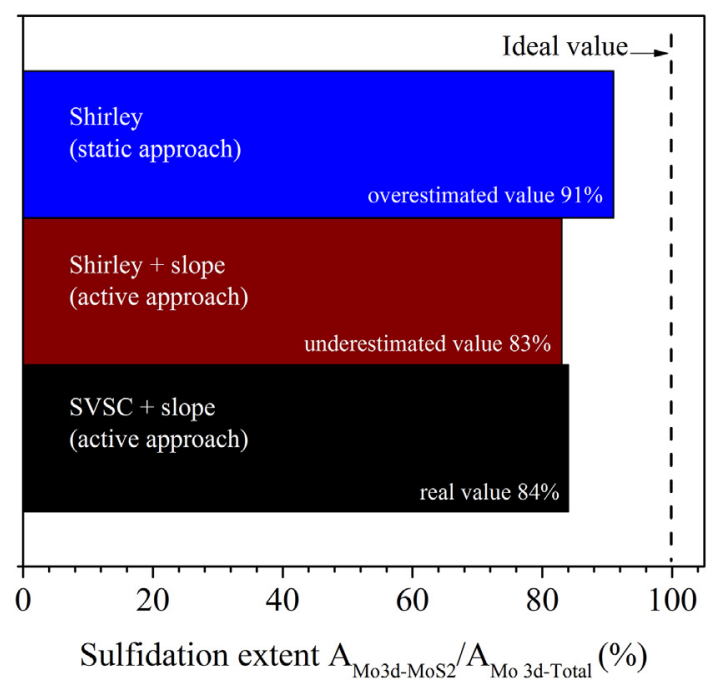

Fig. 5. Values of sulfidation extent $\left(\mathrm{A}_{\mathrm{Mo3d}-\mathrm{Mos} 2} / \mathrm{A}_{\mathrm{Mo3d}-\mathrm{Total}}\right)$ obtained with the three types of background compared in this work: Shirley (static approach), Shirley-Sherwood + slope (active approach) and SVSC + slope (active approach) for the sample CoMoAl. 


\section{Conclusions}

The use of removal background methods SVSC in combination with slope background subtraction under active approach, have been proved accurate models to remove background in cases where discrimination of different contributions of background must be done, as was the case of the overlapped peaks in the regions Mo 3d-S $2 s$ and S 2p-Si 2p. Using the areas obtained with the methods compared here and employing physical parameters, the stoichiometry of $\mathrm{MoS}_{2}$ and $\mathrm{Al}_{2} \mathrm{O}_{3}$ was obtained. It was conclusive that when overlapped peaks are found in the spectrum, distinction of different background strengths must be performed, here allowed by using SVSC background.

This type of analysis is essential for an accurate calculation of the sulfidation extent of the $\mathrm{MoS}_{2}$ active phase $\left(\mathrm{A}_{\text {Mo3d-Mos2 }} / \mathrm{A}_{\text {Mo3d-Total }}\right)$, which is one of the fundamental parameters in the performance of a hydrodesulfurization catalyst. We expect that this work helps the community dedicated to catalysis, to have a tool to calculate accurately the sulfidation extent parameter and have a proper insight for the development of quantitative analysis by XPS.

\section{Declaration of Competing Interest}

There are not conflicts to declare, and the authors declare no competing for financial interests.

\section{Acknowledgements}

Adolfo-Romero Galarza thanks Consejo Nacional de Ciencia y Tecnologia (CONACyT), Mexico for their postdoctoral fellowship (ID. 1908/2010C) . We acknowledge financial support from Direccion General de Asuntos del Personal Academico (DGAPA) Mexico, UNAM, project PAPIIT-IN-113015. The authors appreciate the support provided by Gustavo Gomez-Sosa (CINVESTAV-Qro.) for the acquisition of the XPS data and Alberto Herrera-Gómez (CINVESTAV-Unidad Queretaro, Mexico) for engaging in useful discussion regarding the spectra fitting and for providing computer program AANALYZER version 1.2.

\section{References}

[1] A. Stanislaus, A. Marafi, M.S. Rana, Recent advances in the science and technology of ultra low sulfur diesel (ULSD) production, Catal. Today 153 (2010) 1-68, https:// doi.org/10.1016/j.cattod.2010.05.011.

[2] A. Villarreal, J. Ramírez, L.C. Caero, P.C. Villalón, A. Gutiérrez-Alejandre, Importance of the sulfidation step in the preparation of highly active NiMo/SiO2/ Al2O3 hydrodesulfurization catalysts, Catal. Today 250 (2015) 60-65, https://doi. org $/ 10.1016 / j$.cattod.2014.03.035.

[3] S. Ren, J. Li, B. Feng, Y. Wang, W. Zhang, G. Wen, Z. Zhang, B. Shen, A novel catalyst of Ni,W-surface-Ti-rich-ETS-10/Al2O3: its role and potential of HDS performance for steric hindered sulfur compound 4,6-DMDBT, Catal. Today 263 (2016) $136-140$.

[4] J.A. Toledo-Antonio, M.A. Cortes-Jacome, C. Angeles-Chavez, J. Escobar, M.C. Barrera, E. Lopez-Salinas, Highly active CoMoS phase on titania nanotubes as new hydrodesulfurization catalysts, Appl. Catal. B Environ. 90 (2009) 213-223, https://doi.org/10.1016/j.apcatb.2009.03.024.

[5] V. Costa, B. Guichard, M. Digne, C. Legens, P. Lecour, K. Marchand, P. Raybaud, E. Krebs, C. Geantet, A rational interpretation of improved catalytic performances of additive-impregnated dried CoMo hydrotreating catalysts: a combined theoretical and experimental study, Catal. Sci. Technol. 3 (2013) 140, https://doi.org/10. 1039/c2cy20553j.

[6] C. Bara, A.F. Lamic-Humblot, E. Fonda, A.S. Gay, A.L. Taleb, E. Devers, M. Digne, G.D. Pirngruber, X. Carrier, Surface-dependent sulfidation and orientation of MoS2 slabs on alumina-supported model hydrodesulfurization catalysts, J. Catal. 344 (2016) 591-605, https://doi.org/10.1016/j.jcat.2016.10.001.

[7] L. van Haandel, G.M. Bremmer, E.J.M. Hensen, T. Weber, Influence of sulfiding agent and pressure on structure and performance of $\mathrm{CoMo} / \mathrm{Al} 2 \mathrm{O} 3$ hydrodesulfurization catalysts, J. Catal. 342 (2016) 27-39, https://doi.org/10.1016/j. jcat.2016.07.009.

[8] P. Kumar, M. Singh, R.K. Sharma, G.B. Reddy, An experimental study: role of different ambient on sulfurization of MoO3 into MoS2, J. Alloys Compd. 671 (2016) 440-445, https://doi.org/10.1016/j.jallcom.2016.02.097.

[9] L. Zhang, M. Li, H. Nie, Study on sulfidation degree and morphology of MoS2 catalyst derived from various molybdate precursors, Chin. Pet. Process Pe. Technol. 16 (2014) 1-7.

[10] T. Weber, J.C. Muijsers, J.H.M.C. van Wolput, C.P.J. Verhagen, J.W. Niemantsverdriet, Basic reaction steps in the sulfidation of crystalline $\mathrm{MoO} 3$ to MoS2, as studied by X-ray photoelectron and infrared emission spectroscopy, J. Phys. Chem. 100 (1996) 14144-14150, https://doi.org/10.1021/JP961204Y.

[11] L. Qiu, G. Xu, Peak overlaps and corresponding solutions in the X-ray photoelectron spectroscopic study of hydrodesulfurization catalysts, Appl. Surf. Sci. 256 (2010) 3413-3417, https://doi.org/10.1016/j.apsusc.2009.12.043.

[12] J. Escobar, M.C. Barrera, A.W. Gutiérrez, M.A. Cortés-Jacome, C. Angeles-Chávez, J.A. Toledo, D.A. Solís-Casados, Highly active P-doped sulfided NiMo/alumina HDS catalysts from Mo-blue by using saccharose as reducing agents precursor, Appl. Catal. B Environ. 237 (2018) 708-720, https://doi.org/10.1016/j.apcatb.2018.06 034.

[13] J. Xu, T. Huang, Yu. Fan, Highly efficient NiMo/SiO2-Al2O3 hydrodesulfurization catalyst prepared from germini surfactant-dispersed Mo precursor, Appl. Catal. B Environ. 203 (2017) 839-850, https://doi.org/10.1016/j.apcatb.2016.10.078.

[14] P. Beccat, P. Da Silva, Y. Huiban, S. Kasztelan, Quantitative Surface Analysis by XPS: Application to Hydrotreating Catalysts, 54 (1999), pp. 487-496.

[15] A. Herrera-Gomez, M. Bravo-Sanchez, F.S. Aguirre-Tostado, M.O. Vazquez-Lepe, The slope-background for the near-peak regimen of photoemission spectra, J. Electron Spectrosc. Relat. Phenom. 189 (2013) 76-80, https://doi.org/10.1016/j. elspec.2013.07.006.

[16] A. Herrera-Gomez, M. Bravo-Sanchez, O. Ceballos-Sanchez, M.O. Vazquez-Lepe, Practical methods for background subtraction in photoemission spectra, Surf. Interface Anal. 46 (2014) 897-905, https://doi.org/10.1002/sia.5453.

[17] A. Herrera-Gomez, Robust data analysis, (n.d.) http://rdataa.com. http://www. rdataa.com/.

[18] J.E. Castle, A. Proctor, A.M. Salvi, Curve-fitting in XPS Using Extrinsic and Intrinsic Background Structure, 106 (2000), pp. 65-80.

[19] J.T. Grant, Methods for Quantitative Analysis in XPS.Pdf, 14 (1989), pp. 271-283.

[20] A. Herrera-Gomez, Effect of monochromator X-ray Bragg reflection on photoelectric cross section, J. Electron Spectrosc. Relat. Phenom. 182 (2010) 81-83, https://doi. org/10.1016/j.elspec.2010.07.009.

[21] P.M.A. Sherwood, Introduction to studies of aluminum and its compounds by XPS, Surf. Sci. Spectra 5 (1998) 1-3, https://doi.org/10.1116/1.1247880.

[22] J.T. Kloprogge, L.V. Duong, B.J. Wood, R.L. Frost, XPS study of the major minerals in bauxite: gibbsite, bayerite and (pseudo-)boehmite, J. Colloid Interface Sci. (2006), https://doi.org/10.1016/j.jcis.2005.09.054.

[23] A.M. Venezia, R. Bertoncello, G. Deganello, P. Naturali, V. Archirafi, X-ray photoelectron spectroscopy investigation of pumice-supported nickel catalyst, Surf. Interface Anal. 23 (1995) 239-241.

[24] Y.S. Jin, Q.J. Yan, Z.R. Yin, Y. Chen, Secondary ion mass spectrometry and X-ray photoelectron spectroscopy of $\mathrm{Na}, \mathrm{MoO}, / \mathrm{SiO}$, catalysts for methane oxidative coupling, J. Chem. Soc. Faraday Trans. 91 (1995) 381-384, https://doi.org/10. 1039/ft9959100381. 